\title{
Research Paper \\ Psychometric Properties of the Persian Version of Perceptions of Physical Restraint Use Questionnaire
}

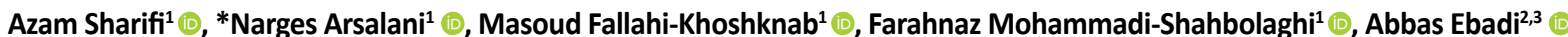

1. Department of Nursing, University of Social Welfare and Rehabilitation Sciences, Tehran, Iran

2. Behavioral Sciences Research Center, Life Style Institute, Baqiyatallah University of Medical Sciences, Tehran, Iran.

3. Department of Nursing Management, School of Nursing, Baqiyatallah University of Medical Sciences, Tehran, Iran.

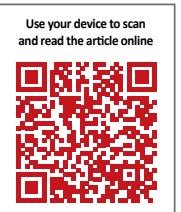

Citat on: Sharifi A, Arsalani N, Fallahi-Khoshknab M, Mohammadi-Shahbolaghi F, Ebadi A. [Psychometric Properties of the Persian Version of Perceptions of Physical Restraint Use Questionnaire (Persian)]. Iranian Journal of Ageing. 2021; 16(2):260273. https://doi.org/10.32598/sija.16.2.2855.1

\section{(i) (8)}

Received: 03 Dec 2019

Accepted: 11 May 2020

Available Online: $01 \mathrm{Jul} 2021$

Key words:

Physical restraint, Factor analysis, Older People, Nurse

\section{A B STRACT}

Objectives One of the fundamental responsibilities of nurses in care is to prevent harm to the patients and others. Many nurses use physical restraints to achieve this goal. Considering that the use of physical restraints in older patients is associated with physical and psychological complications and ethical issues, it is important to identify the nurses' perceptions of using physical restraint in hospitalized older adults. This study aims to evaluate the psychometric properties of the Persian version of Perception of Physical Restraint Use Questionnaire (PRUQ) to be used in caring for hospitalized older adults in Iran.

Methods \& Materials This is a methodological study with a descriptive cross-sectional design that was conducted in hospitals in Kermanshah (Western Iran) in 2019. After obtaining permission from the developers of PRUQ, translation to Persian was performed according to standard protocol of World Health Organization. Then, the Persian version was completed by 330 nurses who were selected by a convenience sampling method based on the inclusion criteria. Then, its face validity and content validity (qualitatively and quantitatively), construct validity using Exploratory Factor Analysis (EFA) and Confirmatory Factor Analysis (CFA), test-retest reliability, internal consistency and structural reliability were assessed. Statistical analysis was performed in SPSS v. 23 and AMOS v. 5 applications.

Results Results showed a Content Validity Index (CVR) of 0.78 and a scale CVI of 0.88 . Based on the results of EFA and CFA, three factors were extracted including "prevention of fall", "prevention of interruption or interference in treatment" and "providing safe environment". The good fit of the three-factor model of the Persian PRUQ was approved based on the model fit indices (Chi square/degree of freedom=2.27, Goodness-of-Fit Index $=0.83$, Comparative Fit Index $=0.91$, Normed Fit Index $=0.92$, Root Mean Square Error of Approximation=0.08). The reliability of the questionnaire was confirmed by Cronbach's alpha coefficient (0.82), structural reliability (>0.70) and intraclass correlation coefficient (0.86).

Conclusion The Persian version of PRUQ has good validity and reliability to be used in nurses working in hospitals in Iran. It can be used in future studies in this population.

\section{* Corresponding Author: \\ Narges Arsalani, PhD.}

Address: Department of Nursing, University of Social Welfare and Rehabilitation Sciences, Tehran, Iran.

Tel: +98 (912) 3702951

E-mail: nargesarsalani@gmail.com 


\section{Extended Abstract}

\section{Introduction}

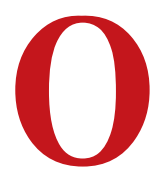

ne of the most important responsibilities of nurses in care is to prevent harm to the patient and others, and many nurses use physical restraint to achieve this goal [4]. Physical restraint is any device and material that is attached or adjacent to patient's body such that $\mathrm{s} /$ he cannot control or easily remove it. Ankle or wrist restraints, chairs with table trays, or full use of bedside rails are some of physical restraints [5]. Considering that the use of physical restraints in older patients is associated with physical and psychological complications and ethical issues [6-8], identifying nurses' perceptions of using physical restraints on the hospitalized older adults is of particular importance $[15,16,19-22]$. This study aims to determine the psychometric properties of the Persian version of the Perceptions of Restraint Use Questionnaire (PRUQ).

\section{Methods}

This is a methodological study with a descriptive crosssectional design that was conducted in 2019 in hospitals of Kermanshah city (West Iran). After obtaining ethical approval from the ethics committee of the University of Social Welfare and Rehabilitation Sciences (Code: IR.USWR. REC.1398.121) and obtaining permission from the developers of PRUQ, the questionnaire was translated into Persian according to the standard protocol of the World Health Organization and using the Forward-Backward method [31]. Then, face validity and content validity of the Persian PRUQ were evaluated qualitatively and quantitatively. To examine the construct validity of the Persian PRUQ, Exploratory Factor Analysis (EFA) and Confirmatory Factor (CFA) were used using a sample size of 330 patients. For EFA, the Persian PRUQ was completed by 170 nurses who were selected by a convenience sampling method based on the inclusion criteria (having at least one year of experience in adult intensive care, internal medicine and surgery, active involvement in decision-making to use physical restraint in hospitalized elderly, and consent to participate in the study).

Kaiser-Meyer-Olkin (KMO) index and Bartlett's test of sphericity were used for EFA. For CFA, 160 nurses were examined using multivariate techniques were used to evaluate the relationships. To fit the model, goodness of fit indices $\left(\chi^{2}, \chi^{2} / \mathrm{df}\right.$, RMSEA, GIF, NFI and CFI) were used. In order to examine the test-retest reliability using the Intraclass Correlation Coefficient (ICC), 30 nurses were selected and were asked to answer the questions two times with a two-week interval. The correlation between the scores of the two tests was determined using the ICC. Internal consistency was calculated using Cronbach's alpha for the whole questionnaire and for each item. Moreover, the reliability of the constructs was examined by examining the ratio of variance in the observed variables to the variance of latent variables in the CFA. Statistical analyzes were performed in SPSS v. 23 and AMOS v. 5 software.

\section{Results}

Out of 330 nurses, $74.2 \%(n=245)$ were women and $63.9 \%(n=211)$ had a bachelor's degree in nursing. Their Mean \pm SD of age and work experience were $36.52 \pm 4.23$ years and $8.22 \pm 4.86$ years, respectively. In assessing qualitative face validity, one item (item 5) needed to be revised to remove ambiguity. In quantitative face validity evaluation, the effect size of all items was higher than 1.5. In qualitative content validity evaluation, the suggestions of geriatricians in terms of clarity and simplicity were applied, in addition to minor grammar corrections. In assessing quantitative content validity, a Content Validity Ratio (CVR) of 0.78 for the entire questionnaire and a scale CVR of 0.88 were obtained. Accordingly, all tool items were accepted. In construct validity evaluation, hidden factors were extracted based on the results of EFA. KMO test result for sample size adequacy was 0.672 which was acceptable and the result of Bartlett's test of sphericity was statistically significant $(\mathrm{P}<0.001)$.

In EFA using maximum likelihood method, Promax rotation and Gravel diagram, three factors "prevention of fall", "prevention of interruption or interference in treatment" and "providing a safe environment" were extracted. These three factors had eigenvalues of 3.58, 3.07 and 2.22, respectively, and explained $52.16 \%$ of the total variance of Persian PRUQ subscales. The results of CFA also provided a good estimate based on the fit indices of the model $\left(\chi^{2} / \mathrm{df}=2.27\right.$, GFI $=$ $0.83, \mathrm{CFI}=0.91, \mathrm{NFI}=0.92, \mathrm{RMSEA}=0.08)$. In the final model of the factor structure of the Persian PRUQ, the variables showed a high correlation with their respective factor. Moreover, there was a correlation between the measurement error in the items related to pulling out a catheter and a feeding tube ( $a 6$ and b6) and that of items related to prevention of breaking open sutures and removing a dressing (d6 and e6). The reliability of the Persian PRUQ was reported acceptable using Cronbach's alpha (0.82), structural reliability $(>0.70)$ and $\mathrm{ICC}=0.86$ (95\% CI: 0.74-0.93, $\mathrm{P}<0.001)$.

\section{Discussion and Conclusion}

The Persian version of PRUQ has acceptable validity and reliability. Due to the small number of items, using this questionnaire is easy and requires little time. Therefore, this 
questionnaire can be used to study and evaluate the perception of the use of physical restraint for geriatric care in Iran and compare the results with the studies conducted in other countries. This questionnaire can also be a good tool for evaluating interventions in the field of physical restraint of the elderly; by comparing the perception of using physical restraint before and after receiving various interventions, it is possible to realize the effectiveness of the interventions to a large extent. Identifying nurses' perceptions of using physical restraint in the elderly can be helpful in planning and policy-making to reduce the use of physical restraints and improve the quality of care for the hospitalized older people.

\section{Ethical Considerations}

\section{Compliance with ethical guidelines}

The Research Ethics Committee of the University of Social Welfare and Rehabilitation Sciences approved the study (Code: IR.USWR.REC.1398.121). All ethical principles are considered in this article. The participants were informed about the purpose of the research and its implementation stages. They were also assured about the confidentiality of their information and were free to leave the study whenever they wished, and if desired, the research results would be available to them.

Funding

This study was extracted from a $\mathrm{PhD}$. dissertation of the first author at the Department of Nursing, University of Social Welfare and Rehabilitation Sciences, Tehran.

\section{Authors' contributions}

All authors equally contributed to preparing this article.

\section{Conflicts of interest}

The authors declare no conflict of interest 


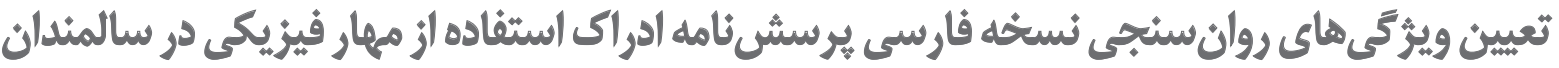

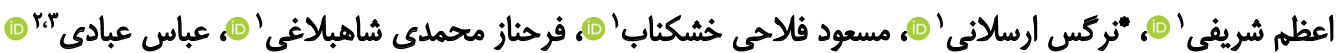

1. بروه يرستارى، دانشكاه علوم توانيخشى و سلامت اجتماعى، تهران، ايران.

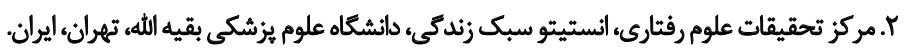

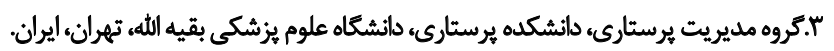

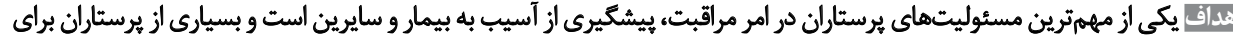

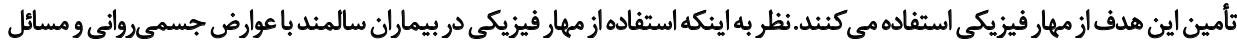

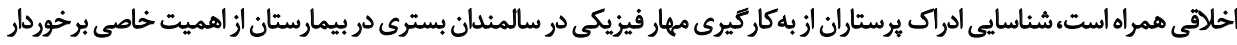

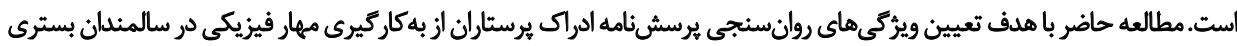
در بيمارستان انجام شد.

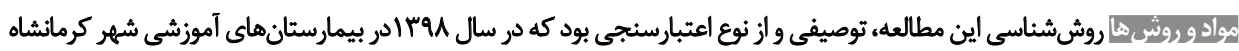

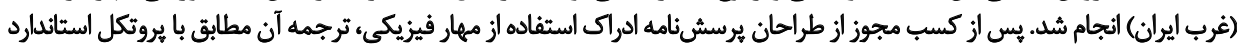

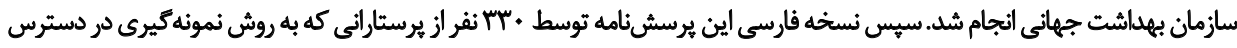

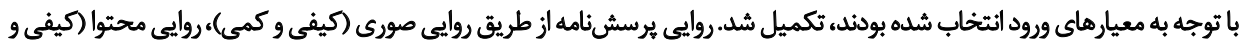

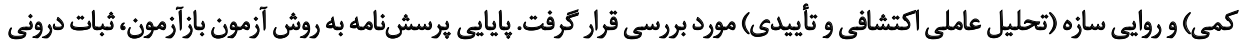

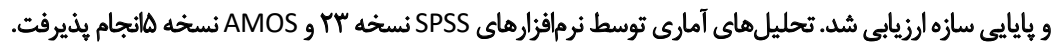

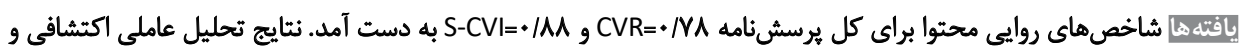

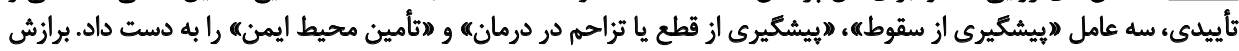

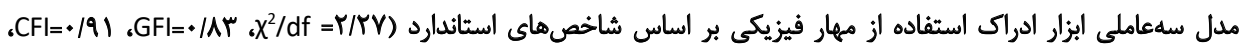

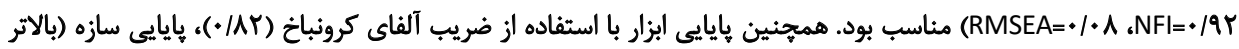

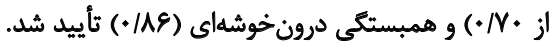

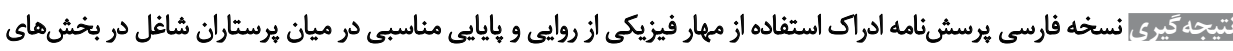

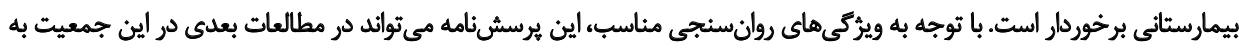

["، []. با وجود اينكه در مورد معنا و تعريف كيفيت مراقبت،

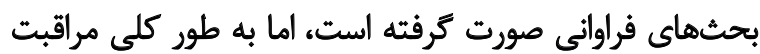

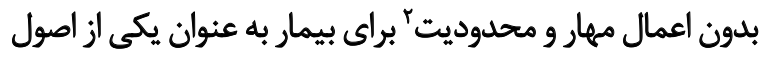

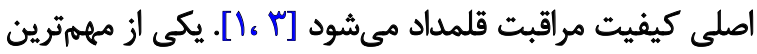

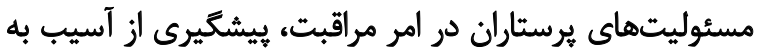

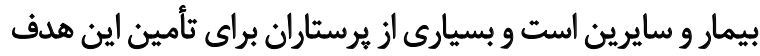

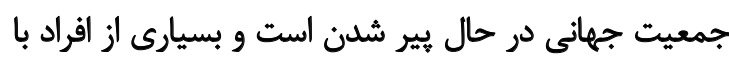

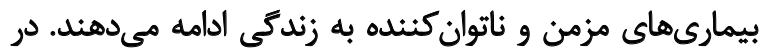

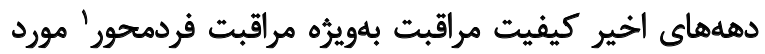

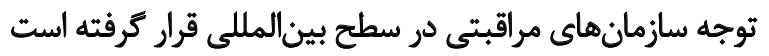

1. Person-Centred Care (PCC)

$$
\text { -.......... }
$$


با هدف افزايش ميزان آكاهي و بهبود عملكرد يرستاران براي كاهش

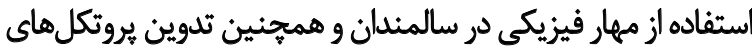

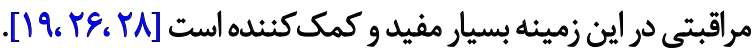

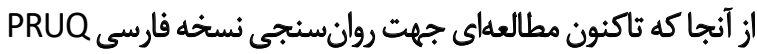

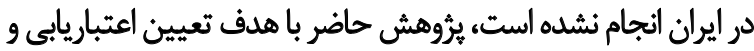
روانسنجى نسخه فارسى اين برسش برونامه انجام شد.

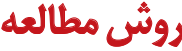

يرؤوهش حاضر از نوع روششئاختى و اعتبارسنجى با طرح توصيفىمقطعى است كه از ارديبهشت تا آبان ماه سال

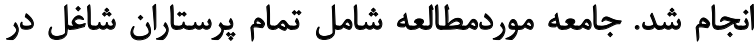

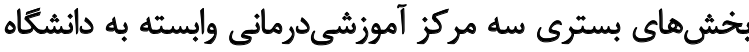

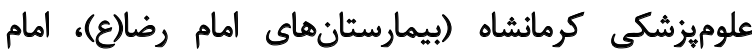

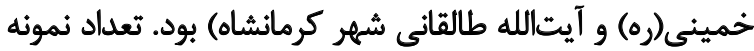

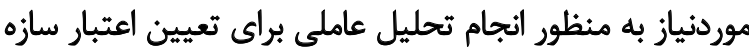

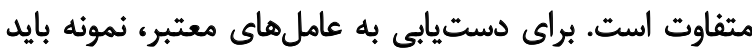

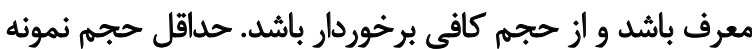

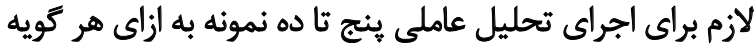

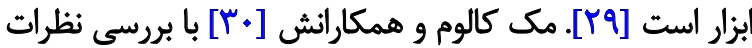

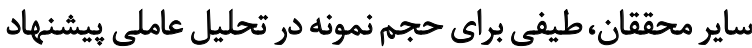

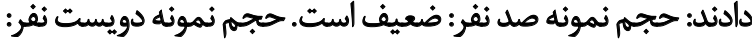

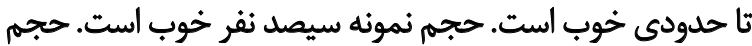

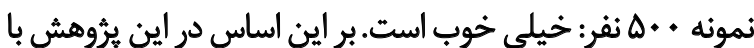

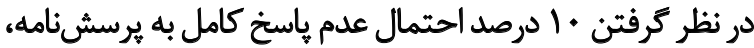

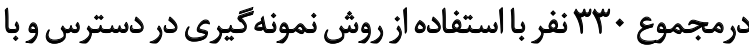

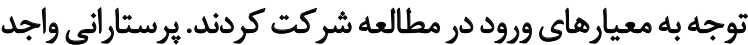

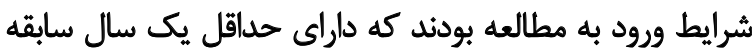

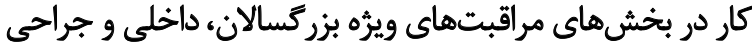

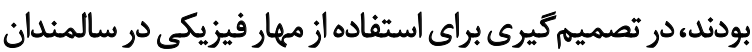

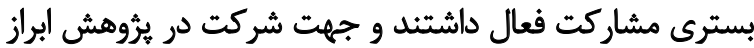

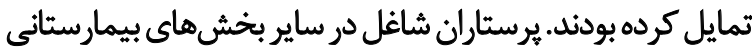

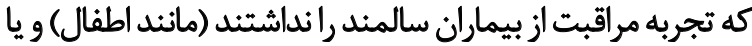

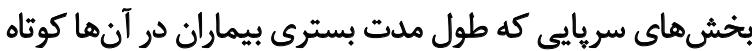

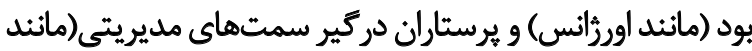

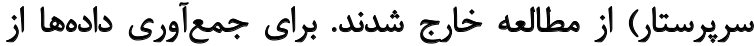

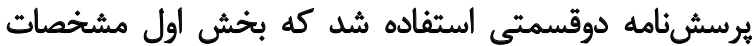

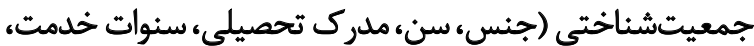

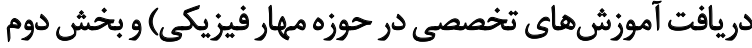

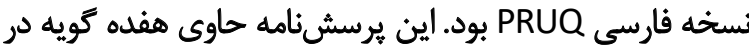

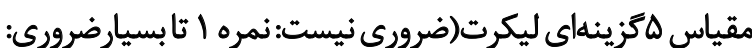

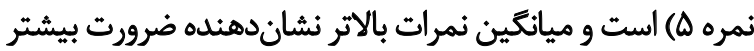

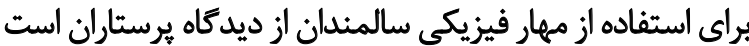
[YF. MT]

در ابتدا يس از مكاتبه با سازندكان يرسشنامه و كسب مجوز

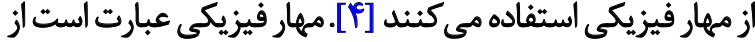

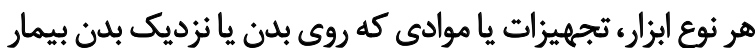

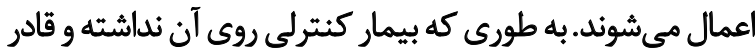

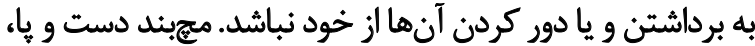

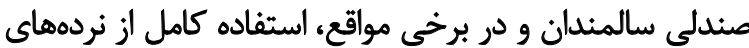

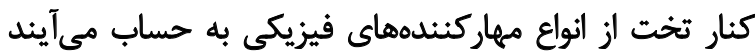

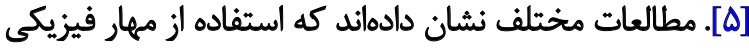

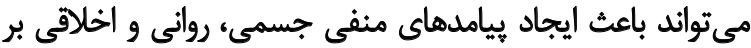

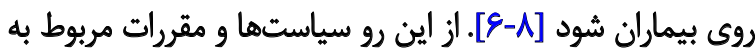

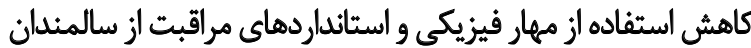

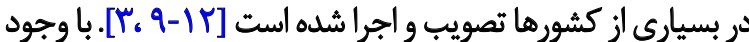

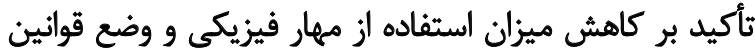

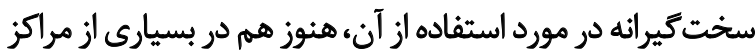

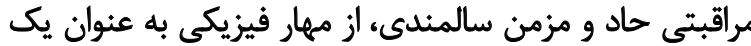

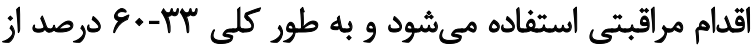

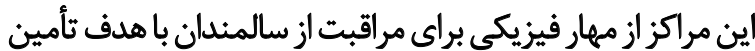

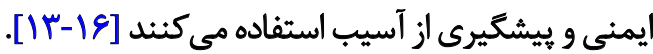
استفاده از مهار فيزيكى يك امر بيجيجيده و اثركذار بر كيفيت

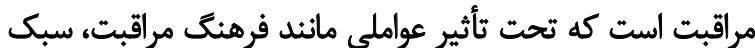

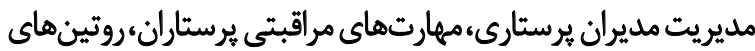

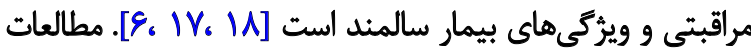

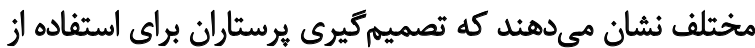

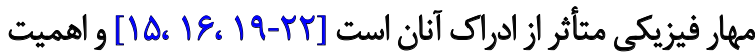

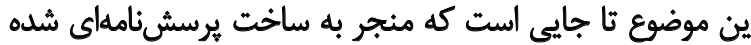

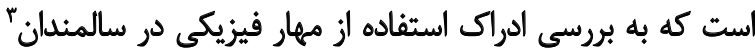

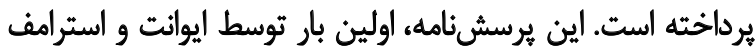

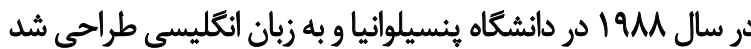

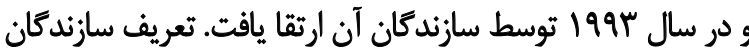

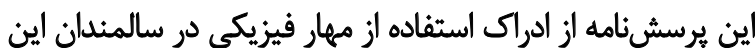

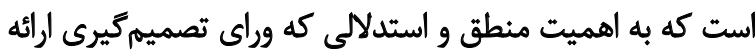

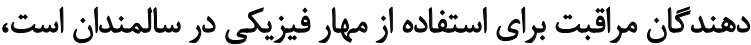

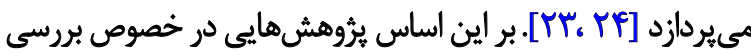

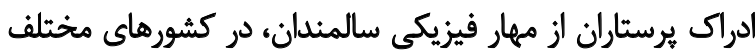

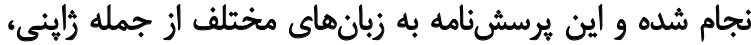

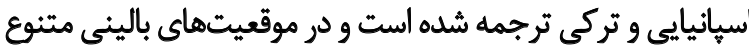

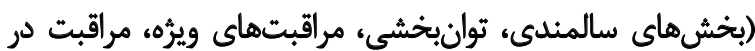

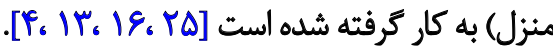
مطالعات نشان مى دهند كه شناسايى ادراك يرستاران ازئ مهار

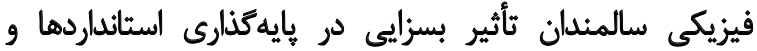

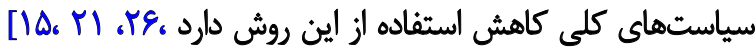

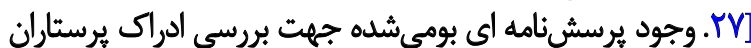

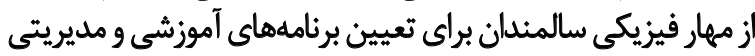


كمى، از دو شاخص نسبت روايى محتواه و ايندكس روايى محتواء

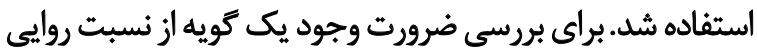

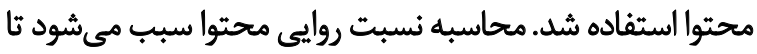

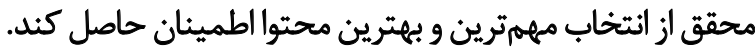

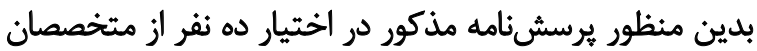

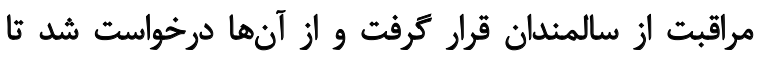

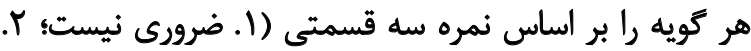

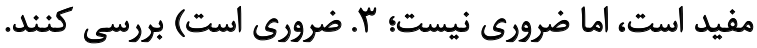

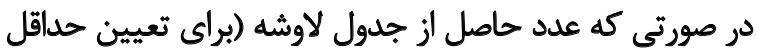

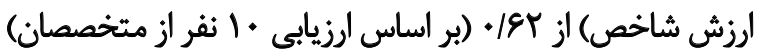

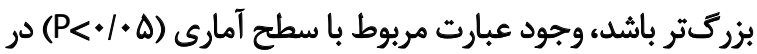

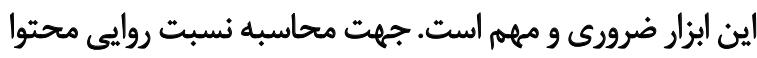

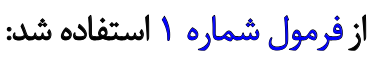

$$
C V R=\frac{n E-N / 2}{N / 2}
$$

در فرمول بالا ne تعداد متخصصانى است كه نمره "ضرورى است" را داده اند و N تعداد كل متخصصان است. براي بررسى مرتبط بودن آيتمها از ايندكس روايى محتوا بر بر بادي

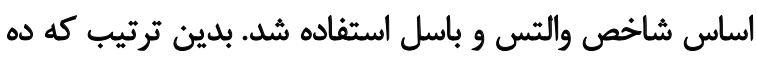

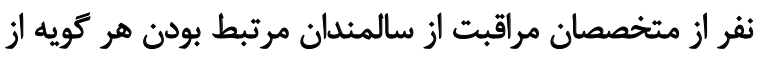

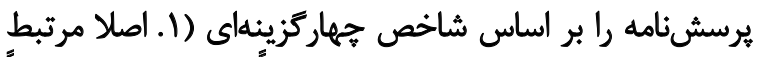

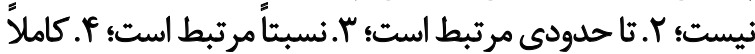

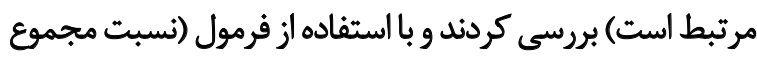

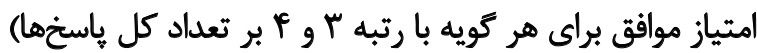

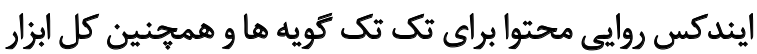

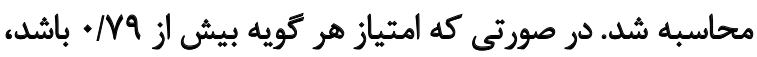

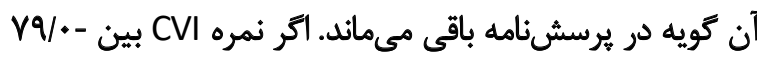

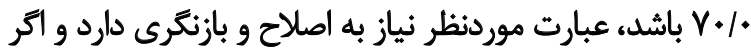

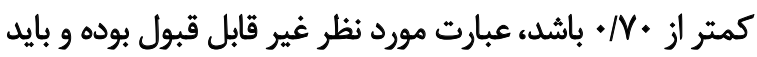

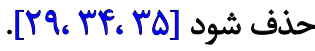

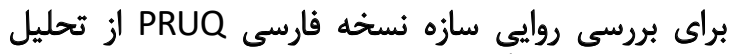

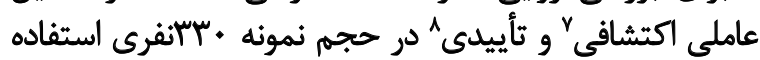

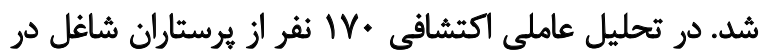

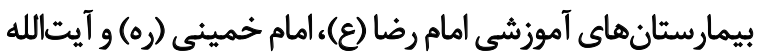

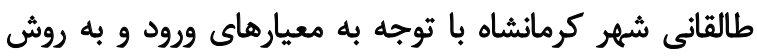

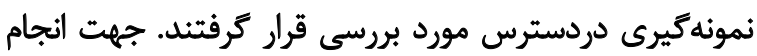
تحليل عاملى اكتشافى از شاخص ميرد مرديز مير اولكين" و آزمون

5. Content Validity Ratio

6. Content Validity Index (CVI)

7. Exploratory Factor Analysis (EFA)

8. Confirmatory Factor Analysis (CFA)

9. Kaiser-Meyer-Olkin (KMO)
استفاده از آن، ترجمه آن مطابق با بروتكل استاندارد سازمان

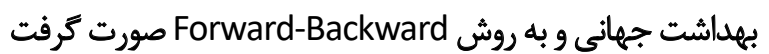

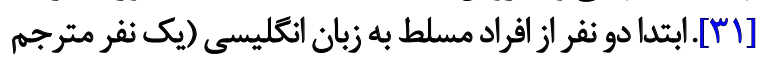

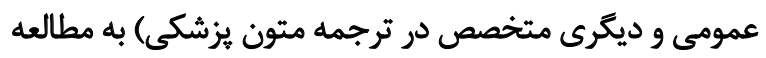

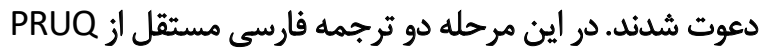

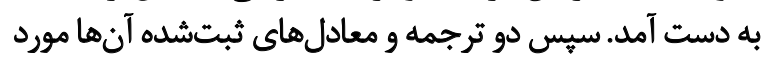

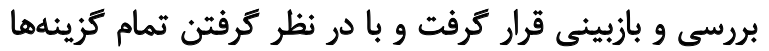

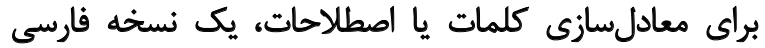

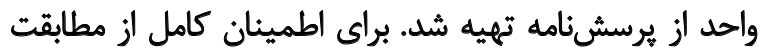

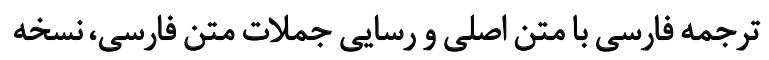

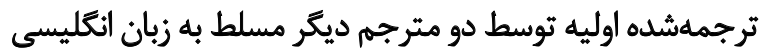

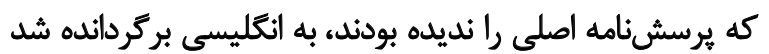

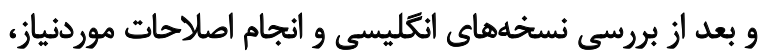

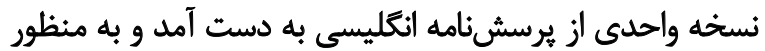

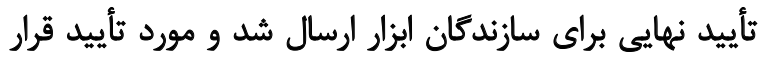

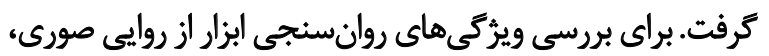
محتواو سازه استفاده شد. بروسي

روايى صورى PRUQ به روش كيفى و كمى صورت كرفت.

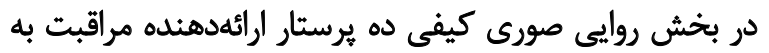

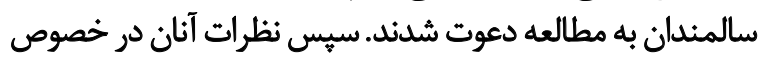

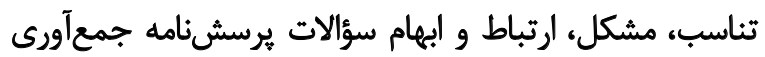

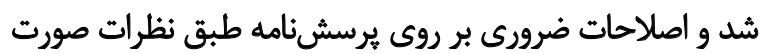

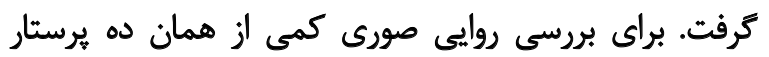

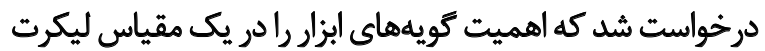

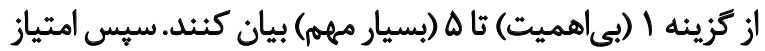

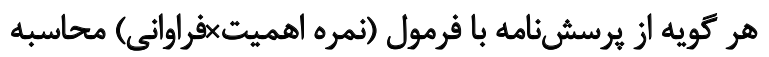

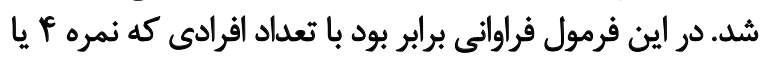

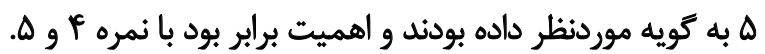

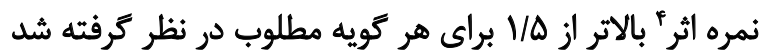

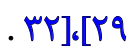

دوايى محتوايى PRUQ نيز به دو روش كيفى و كمى ارزيابى شد.

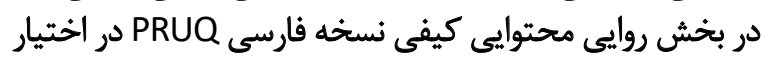

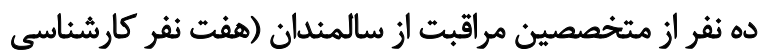

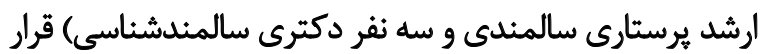

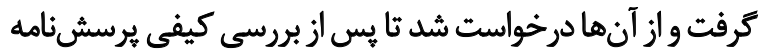

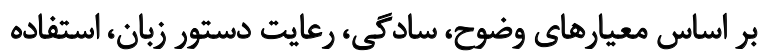

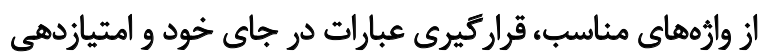

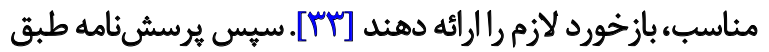

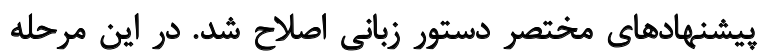
ريزش كويه وجود نداشت. براى بررسى روائى اصلاح محتوا به در اين مرحل 


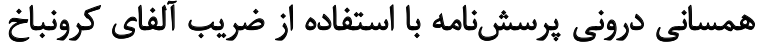

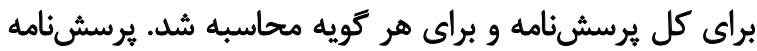

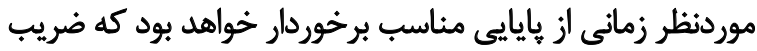

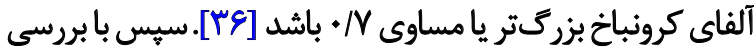

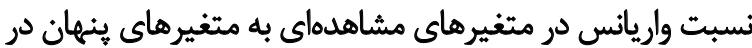

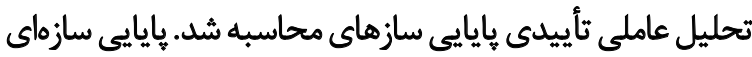

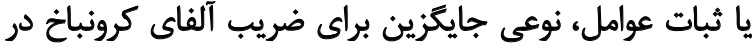

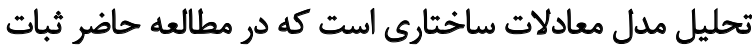

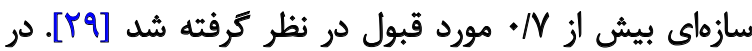

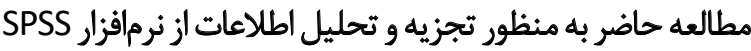
نسخه بr و AMOS نسخه ها ه استفاده شد.

يأفتهها

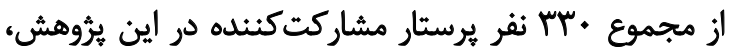

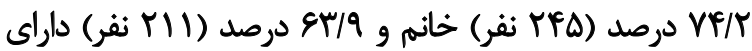

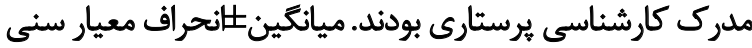

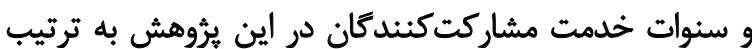

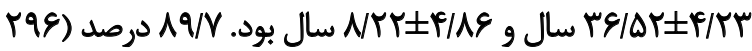

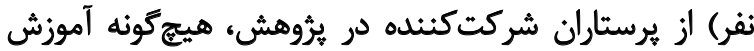
تخصصى در حوزه مهار فيزيكى دريافت نكرده بودند.

در روايى صورى كيفى يك كويه (سؤال ينج) جهت رفع ابهام

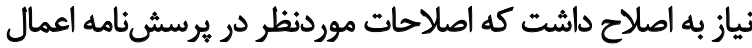

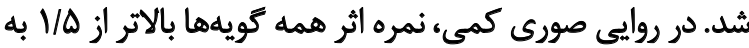

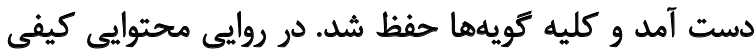

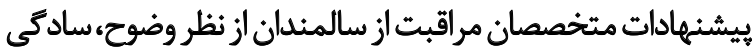

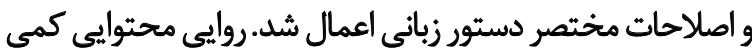

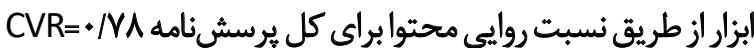

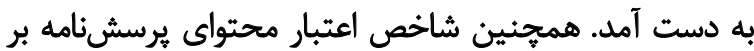

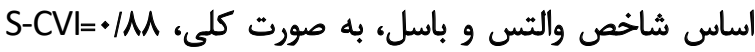

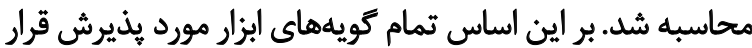

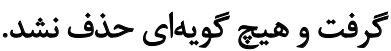

در بررسى روايي سازه، بر اساس نتايج تحليل عاملى اكتشافى دري

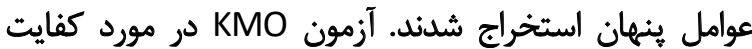

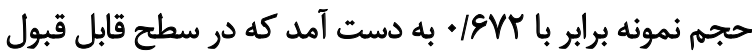

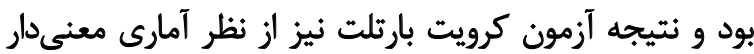

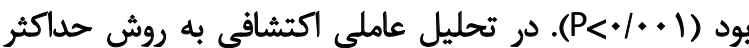

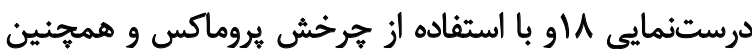

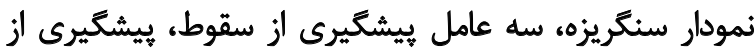

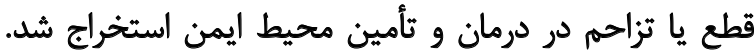

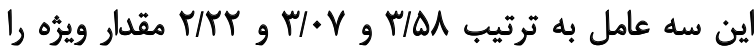

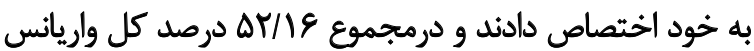
متغيرهاى PRUQ را تبيين كردند (جدول شماره لو ل).
كرويت بارتلت "' استفاده شد. شاخص KMO Kمان نشاندهنده كفايت

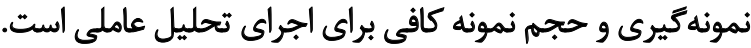

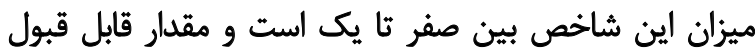

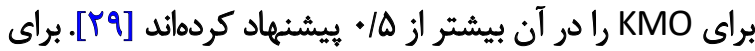

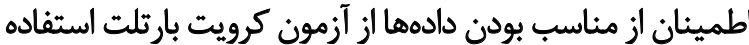

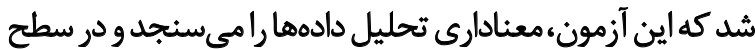

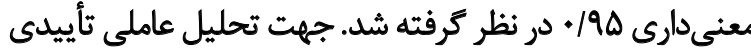

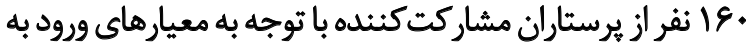

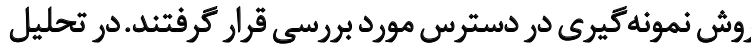

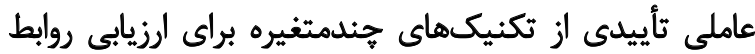

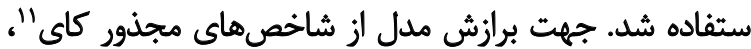

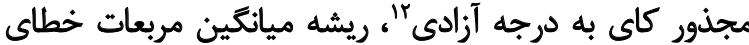

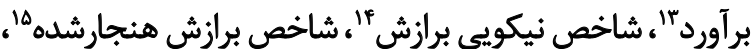

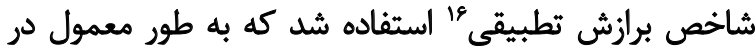

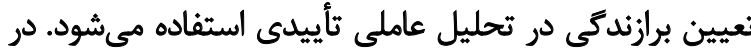

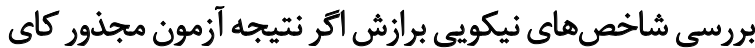

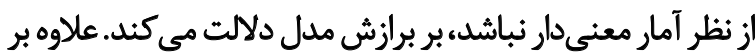

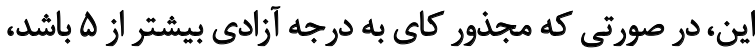

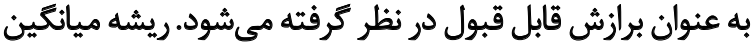

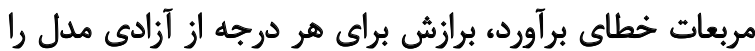

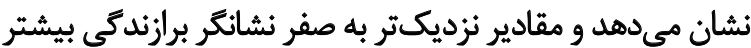

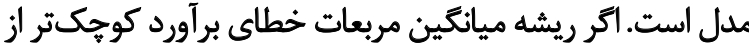

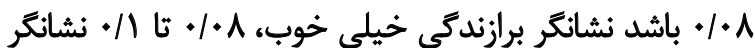

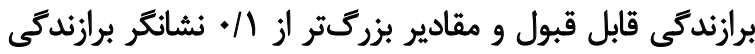

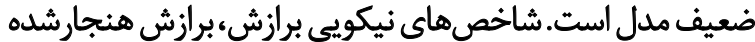

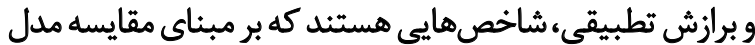

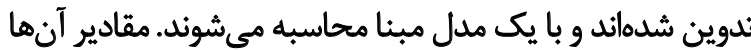

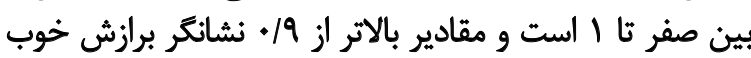

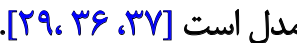

جهت تعيين يايايى به روش آزمون بازآزمون و شاخص

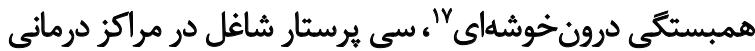

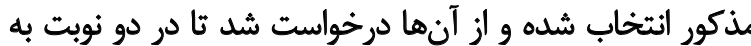

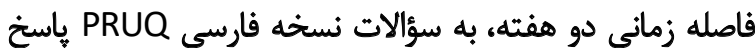

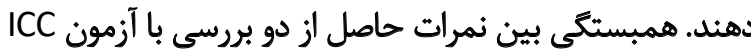

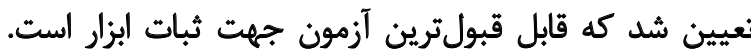

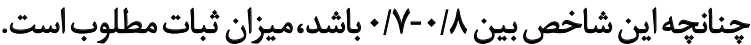

10. Bartlett's Test of Sphericity

11. $\chi^{2}$

12. $\chi^{2} / \mathrm{df}$

13. Root Mean Square Error of Approximation (RMSEA)

14. Goodness of Fit Index (GFI)

15. Normed Fit Index (NFI)

16. Comparative Fit Index (CFI)

17. Interclass Correlation Coefficient (ICC) 
جدول ا. عوامل اكتشافى استخراجشده از نسخه فارسى يرسشنامه ادراك استفاده از مهار فيزيكى

\begin{tabular}{|c|c|c|c|c|}
\hline واريانس & عاملى & & كويه & عامل \\
\hline$r y / \cdot \Delta$ & $\begin{array}{l}. / 9 P \mid \\
. \mid N E T \\
. \mid 811\end{array}$ & 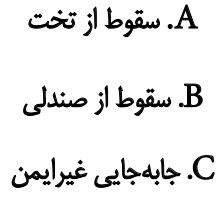 & ا.حفاظت سالمند از & ييشكيرى از سقوط \\
\hline$W \cdot G$ & $\begin{array}{l}. \text { /AVE } \\
. \text { IMA } \\
. / 191 \\
. \text { /NET } \\
. \text { /VAP }\end{array}$ & 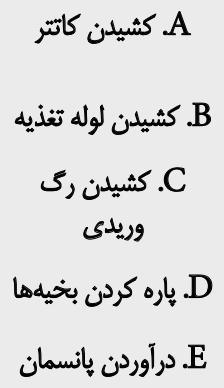 & و ييشكيرى از & تيبزاحيميرى در درمان قطع يا \\
\hline$|r| \cdot \Delta$ & 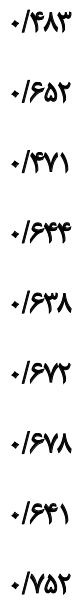 & & 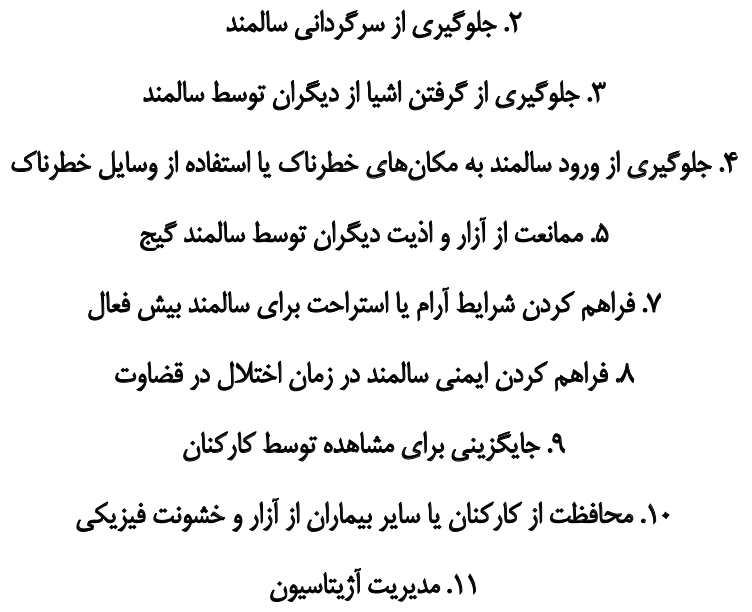 & تامين محيط ايمن \\
\hline
\end{tabular}

جدول r. مقادير شاخصهاى برازش الكوى تحليل عاملى تأييدى يرسشئمه ادراك استفاده از مهار فيزيكى

\begin{tabular}{|c|c|}
\hline مثادير مشاهدهشده & شاخص برازث \\
\hline$r E H / \cdot V$ & مجذور كاى \\
\hline 118 & درجه آزادى \\
\hline .1 .0 & سطع معني دارى \\
\hline$r / T V$ & مجذور كاى به درجه آزادى \\
\hline$+1+\lambda$ & ريشه ميانكين مربعات خطاى برآورد \\
\hline ./Ar & شاخص نيكويي برازش \\
\hline.$/ 97$ & شاخص برازش هنجارشله \\
\hline.$/ 91$ & شاخص برازش تطبيقى \\
\hline
\end{tabular}

L 


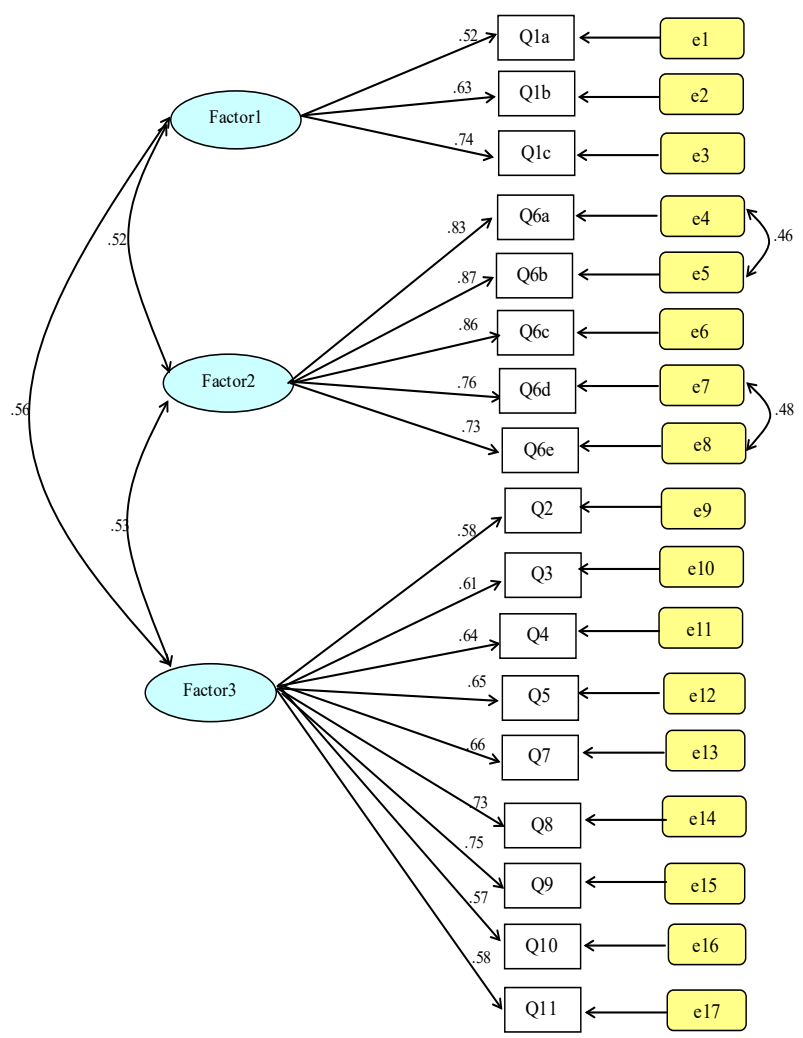

L

در زبان فارسى انجام شد. اين برسشنامه براى ارزيابى ادراى

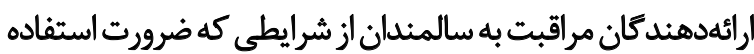

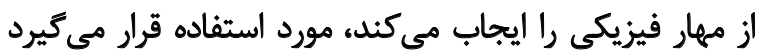

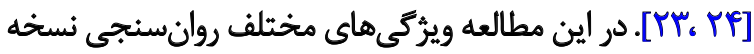

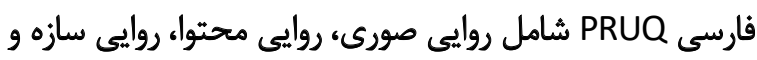

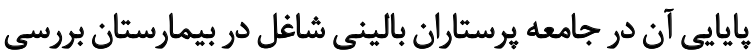

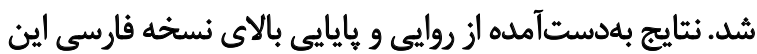
يرسشنامه حمايت مي كند. تاكنون PRUQ به جندين زبان مختلف استانداردسازى شده

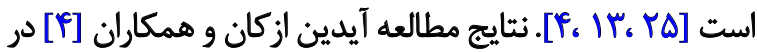

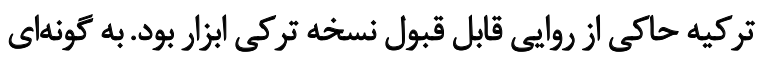

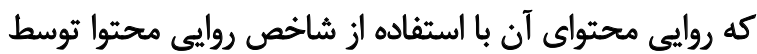

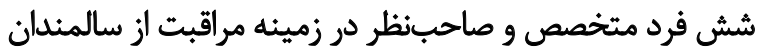

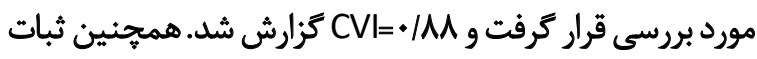

تصوير ا. ساختار نهايى مدل يرسشنامه ادراك استفاده از مهار فيزيكى

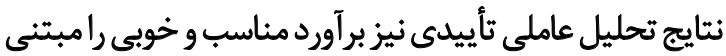

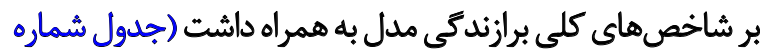

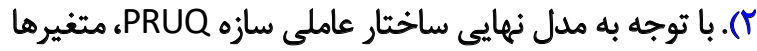

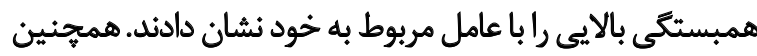

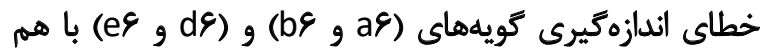
همبستكى داشتند (تصوير شماره (1)).

همسانى درونى كويههاى PRUQ به كمك آلفاى كرونباخ،

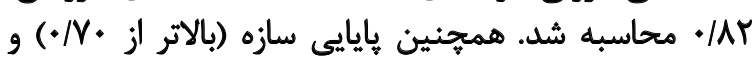

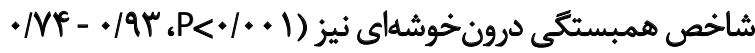
ICC = •/A\& (Cl 9D: بحث اين مطالعه با هدف روانسنجى نسخه فارسى يرسشنامئه ادراك استفاده از مهار فيزيكى در سالمندان و ارائه يك ابزار معتبر فئر

جدول ب. نتايج يايايى با روش همسانى درونى(آلفاى كرونباخ)، هايايى سازه و شاخص همبستكى درونخوشهاى

\begin{tabular}{|c|c|c|c|c|}
\hline ثُشاخص همبستئى درونخوشهاي & هايايي سازه & ألفاي كرونباخ & تعداد كويهها & ابعاد \\
\hline .191 & $\cdot / V A$ &.$/ 9 r$ & $r$ & ييشكيرى از سقوط \\
\hline .119 & - IAT & .118 & $\Delta$ & ييشكيرى از قطع يا تزاحم در درمان \\
\hline - MY & $\cdot M$ & $\cdot M P$ & 9 & ثأمين محيط ايمن \\
\hline
\end{tabular}


نشانكر دارا بودن حداقل ميزان قابل قبولى از بار عاملى بودي

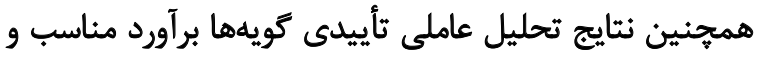

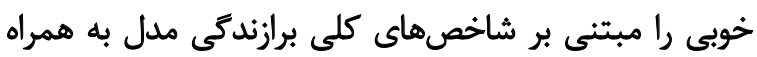

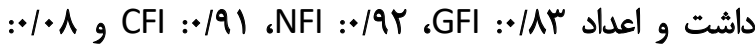

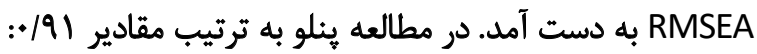

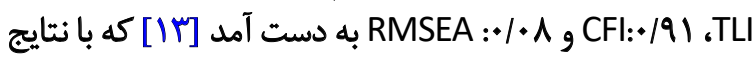

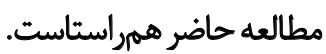

بر اساس مدل نهايى سازه PRUQ ارتباطى بين خطاي

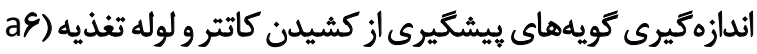

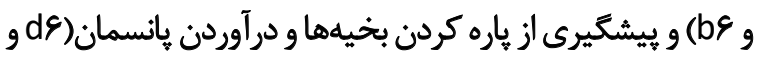

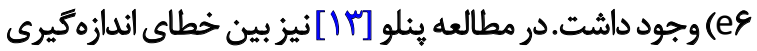

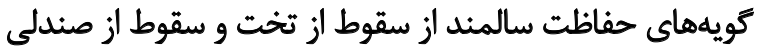

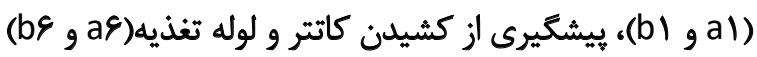

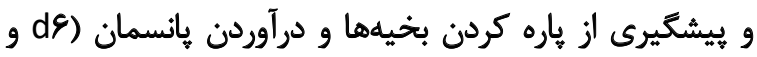

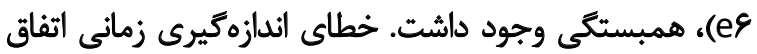

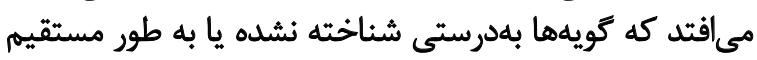

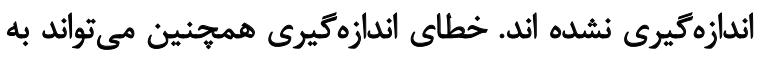

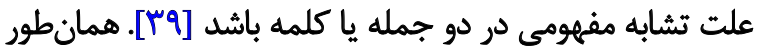

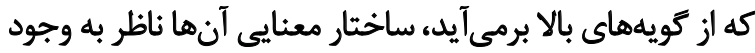

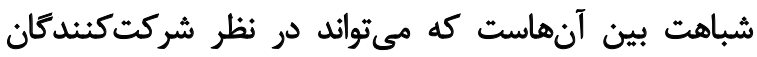

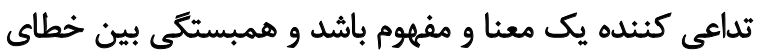

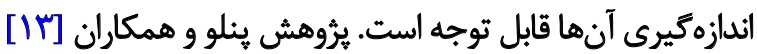

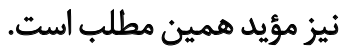

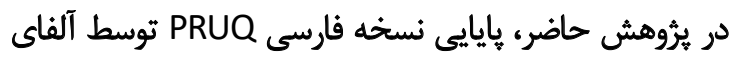

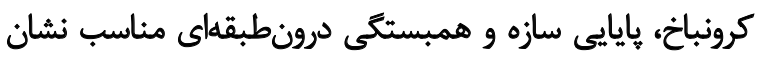

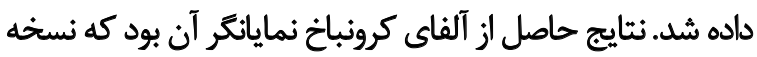

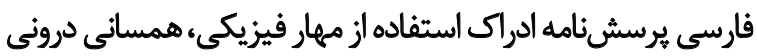

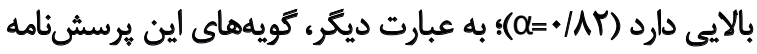

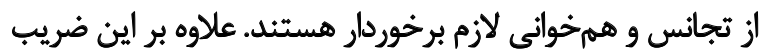

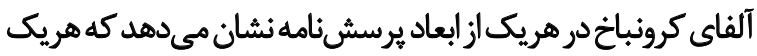

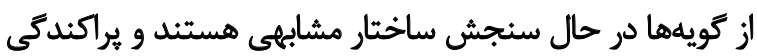

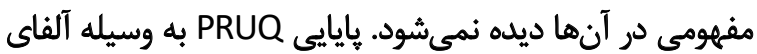

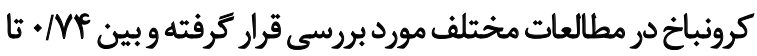

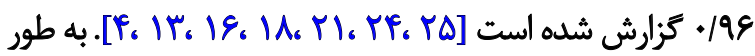

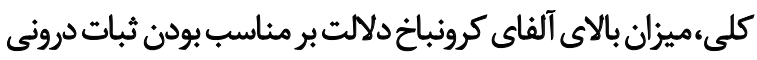

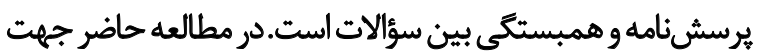

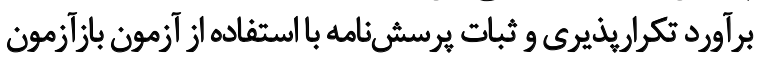

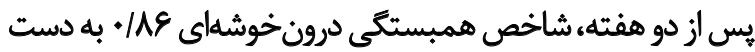

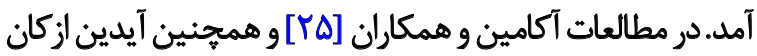

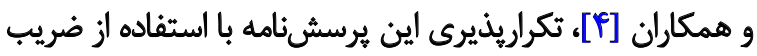

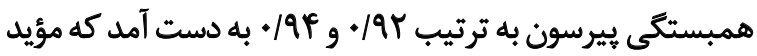
تكراريذيرى اين يرسشنامه برست ترتي
درونى ابزار با استفاده از ضريب آلفاى كرونباخ به/ • كزارش شد

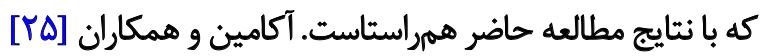

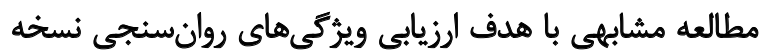

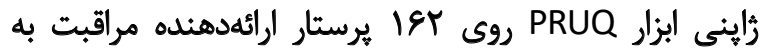

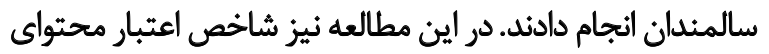
ابزار CVI=|Aه و ثبات درونى ابزار

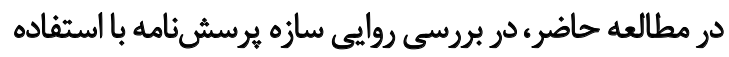

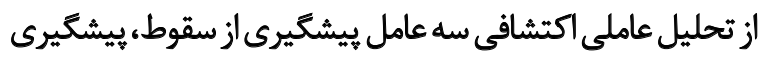

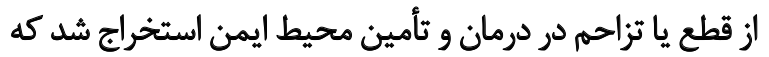

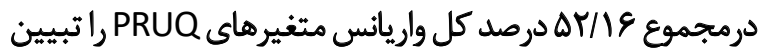

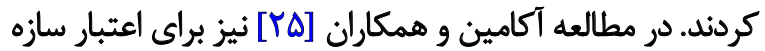

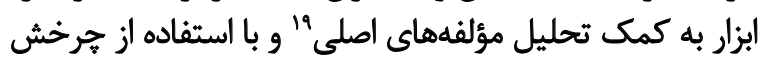

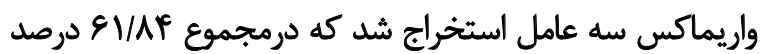

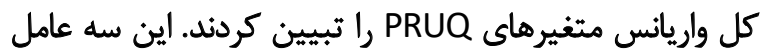
شامل فراهم كردن محيط ايمن براى بيمار و سايرين (9 كويه كريه)،

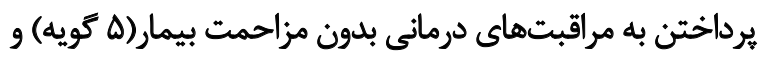

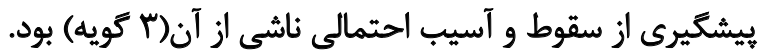

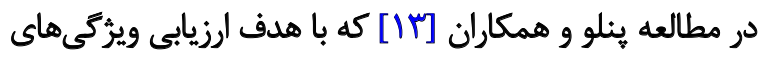

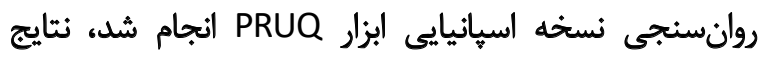
مشابيهى به دست آمد و در تحليل عاملي إئى اكتشافى (با استفاده

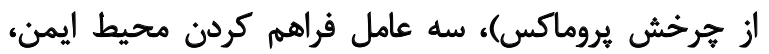

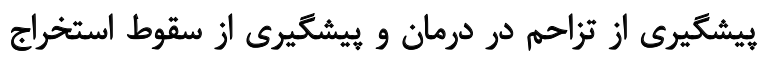

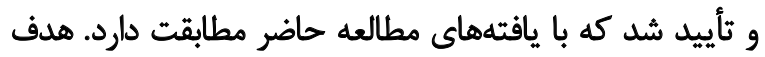

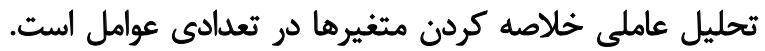

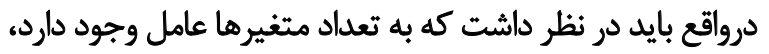

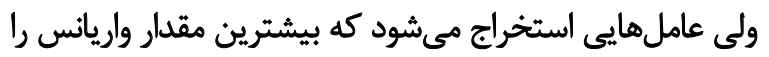

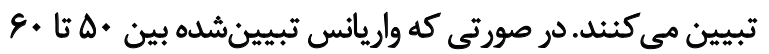

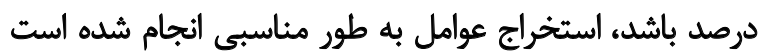

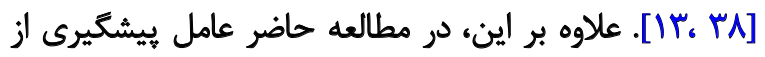

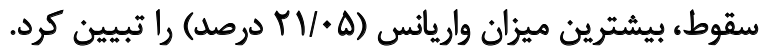

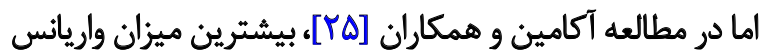

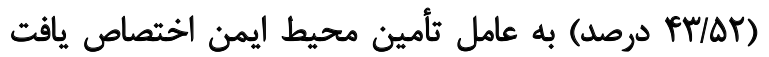

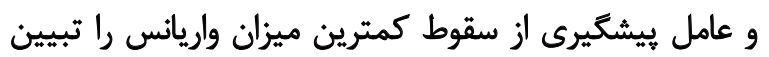

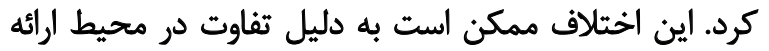

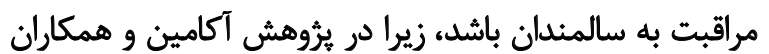

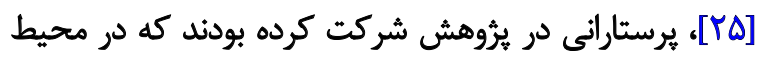

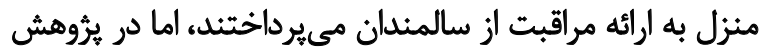

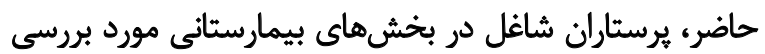

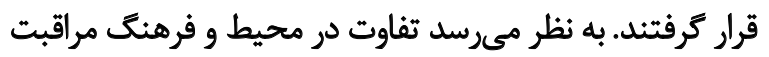

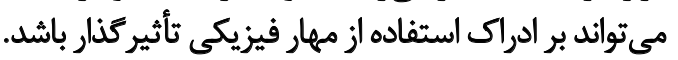
در مطالعه حاضر با توجه به شاخصهاى تزارش أشده، برازش باري

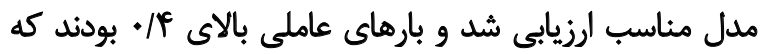




\section{مشاركت نويسند مَان}

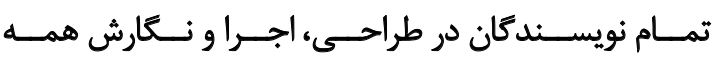

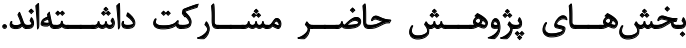

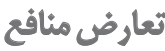

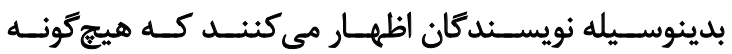

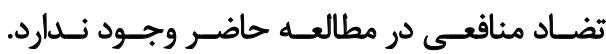

تثيجه تيرى نهايي

نسخه فارسى PRUQ از روايى و هايايى قابل قبولى برخوردار

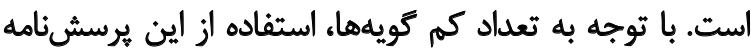

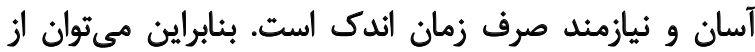

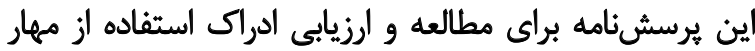

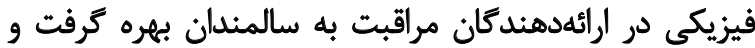

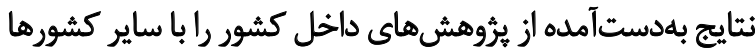

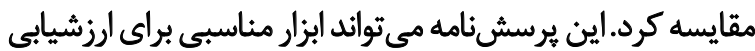

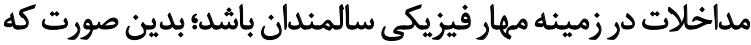

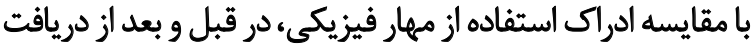

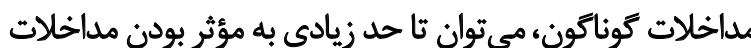

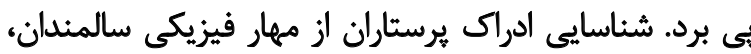

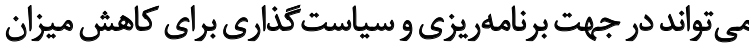

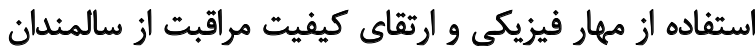

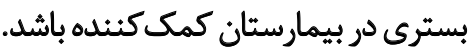

در خايان بايد اضافه كرد كه در اين مطالعه، نمونه ثيروهش

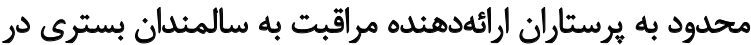

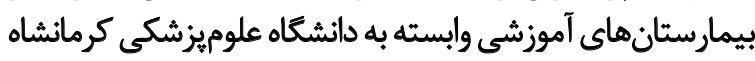

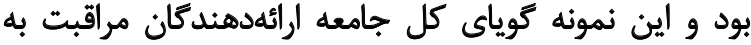

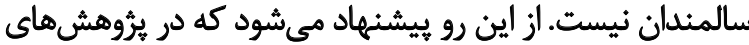

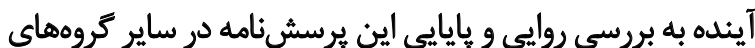

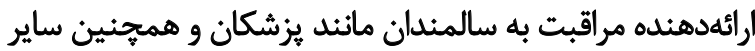

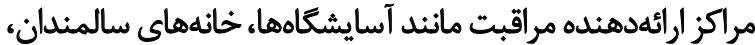

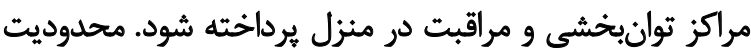

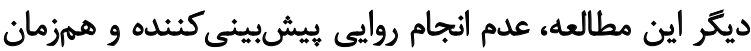

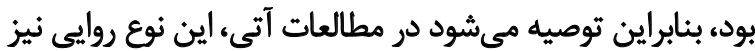

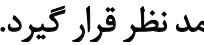

مالاحظات اخلاقي

ييروى أز أصول اخلاق يثوهش

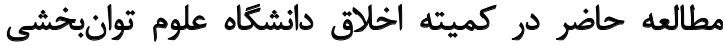

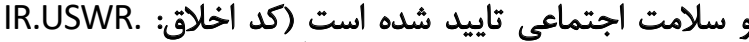

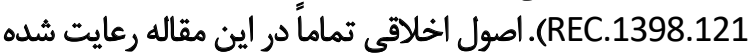

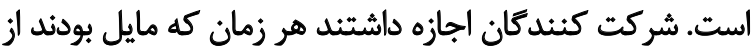

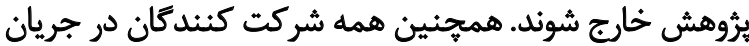

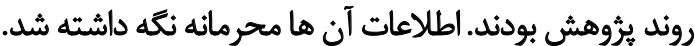

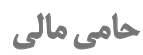

اين يثروهش بخشى از رسالهى دكترى نويسنده اول در كروه

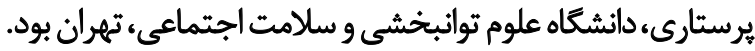




\section{References}

[1] Health Information \& Quality Authority. Guidance for designated centers: Restraint procedures (GDE3), Version 3 [Internet]. 2016 [Updated 2016 April]. Available from: https://www.hiqa. ie/sites/default/files/2017-01/Guidance-on-restraint-procedures.pdf

[2] Centers for Medicare \& Medicaid Services. Medicare and Medicaid programs; Reform of requirements for long-term care facilities (Proposed Rule 80 FR 42167) [Internet]. 2016 [Updated 2016 July 16]. Available from: https://www.federalregister.gov/ documents/2015/07/16/2015-17207/medicare-and-medicaidprograms-reform-of-requirements-for-long-term-care-facilities

[3] Sharifi A, Arsalani N, Fallahi-Khoshknab M, Mohammadi-Shahbolaghi F. The principles of physical restraint use for hospitalized elderly people: An integrated literature review. Systematic Reviews. 2021; 10:129. https://doi.org/10.1186/s13643-021-01676-8

[4] Aydin Özkan S, Karaka T, İster ED. Validity and reliability of the "perceptions of restraint use questionnaire" for use in Turkey. Turkish Journal of Geriatrics. 2017; 20(1):30-7. https:/ / www.researchgate.net/publication/316061120

[5] Bleijlevens MHC, Wagner LM, Capezuti E, Hamers JPH, International Physical Restraint Workgroup. Physical restraints: Consensus of a research definition using a modified Delphi technique. Journal of the American Geriatrics Society. 2016; 64(11):2307-10. [DOI:10.1111/jgs.14435]

[6] Hofmann H, Hahn S. Characteristics of nursing home residents and physical restraint: A systematic literature review. Journal of Clinical Nursing. 2014; 23(21-22):3012-24. [DOI:10.1111/ jocn.12384] [PMID]

[7] Berzlanovich AM, Schöpfer J, Keil W. Deaths due to physical restraint. Deutsches Ärzteblatt International. 2012; 109(3):27-32 [DOI:10.3238/arztebl.2012.0027]

[8] Kong EH, Choi H, Evans LK. Staff perceptions of barriers to physical restraint-reduction in long-termcare: A meta-synthesis. Journal of Clinical Nursing. 2017; 26(1-2):49-60. [DOI:10.1111/ jocn.13418] [PMID]

[9] Hjaltadóttir I, Ekwall AK, Nyberg P, Hallberg IR. Quality of care in Icelandic nursing homes measured with Minimum Data Set quality indicators: Retrospective analysis of nursing home data over 7 years. International Journal of Nursing Studies. 2012; 49(11):1342-53. [DOI:10.1016/j.jinurstu.2012.06.004]

[10] Department of Health Ireland. Towards a restraint free environment in nursing homes [Internet]. 2011 [Updated 2020 October 26]. Available from: https://www.gov.ie/en/publication/ e7f401-towards-a-restraint-free-environment-in-nursing-homes/

[11] Royal College of Nursing. Let's talk about restraint: Rights, risks and responsibility [Internet]. 2008 [Updated 2008]. Available from: https://restraintreductionnetwork.org/wp-content/ uploads/2016/11/Lets-talk-about-restraint.pdf

[12] Cleary KK, Prescott K. The use of physical restraints in acute and long-term care: An updated review of the evidence, regulations, ethics, and legality. Journal of Acute Care Physical Therapy. 2015; 6(1):8-15. [DOI:10.1097/JAT.0000000000000005]

[13] Penelo E, Estévez-Guerra GJ, Fariña-López E. Validity andmeasurement invariance of the Physical Restraint Use Questionnaire (PRUQ) in nursing staff. Journal of Clinical Nursing. 2018; 27(5-6):e1179-88. [DOI:10.1111/jocn.14253] [PMID]
[14] Estévez-Guerra GJ, Fariña-López E, Núñez-González E, Gandoy-Crego M, Calvo-Francés F, Capezuti EA. The use of physical restraints in long-term care in Spain: A multi-center cross-sectional study. BMC Geriatrics. 2017; 17:29. [DOI:10.1186/s12877-0170421-8]

[15] Jiang $\mathrm{H}, \mathrm{Li} \mathrm{Ch}, \mathrm{Gu}$ Y, He Y. Nurses' perceptions and practice of physical restraint in China. Nursing Ethics. 2015; 22(6):652-60. [DOI:10.1177/0969733014557118]

[16] Karaka T, Aydin Özkan S, Derya İster E. Physical restraint use in elderly patients: Perceptions of nurses in university hospitals. Turkish Journal of Geriatrics. 2018; 21(4):588-95. [DOI:10.31086/ tjgeri.2018.66

[17] Goethals S, de Casterlé BD, Gastmans C. Nurses' decision-making process in cases of physical restraint in acute elderly care: A qualitative study. International Journal of Nursing Studies. 2013; 50(5):603-12. [DOI:10.1016/j.ijnurstu.2012.10.006]

[18] Leahy-Warren P, Varghese V, Day MR, Curtin M. Physical restraint: Perceptions of nurse managers, registered nurses and healthcare assistants. International Nursing Review. 2018; 65(3):327-35. [DOI:10.1111/inr.12434]

[19] Kurata S, Ojima T. Knowledge, perceptions, and experiences of family caregivers and home care providers of physical restraint use with home-dwelling elders: A cross-sectional study in Japan. BMC Geriatrics. 2014; 14:39. [DOI:10.1186/1471-2318-14-39]

[20] Hevener S, Rickabaugh B, Marsh T. Using a decision wheel to reduce use of restraints in a medical-surgical intensive care unit. American Journal of Critical Care. 2016; 25(6):479-86. [DOI:10.4037/ajcc2016929]

[21] Fariña-López E, Estévez-Guerra GJ, Polo-Luque ML, Hanzeliková Pogrányivá A, Penelo E. Physical restraint use with elderly patients: Perceptions of nurses and nursing assistants in Spanish acute care hospitals. Nursing Research. 2018; 67(1):55-9. [DOI:10.1097/NNR.0000000000000252]

[22] Fariña-López E, Estévez-Guerra GJ, Gandoy-Crego M, PoloLuque LM, Gómez-Cantorna C, Capezuti EA. Perception of Spanish nursing staff on the use of physical restraints. Journal of Nursing Scholarship. 2014; 46(5):322-30. [DOI:10.1111/jnu.12087]

[23] Strumpf NE, Evans LK. Physical restraint of the hospitalized elderly: Perceptions of patients and nurses. Nursing Research. 1988; 37(3):132-7. [PMID]

[24] Evans LK, Strumpf NE. Frailty and physical restraint. In: Morley JE, Coe RM. Aging and Musculoskeletal Disorders: Concepts, Diagnosis, and Treatment. New York: Springer; 1993. https:// books.google.com/books?id=vBBsAAAAMAAJ\&dq

[25] Akamine Y, Yokota T, Kuniyoshi M, Uza M, Takakura M. Reliability and validity of the Japanese version of physical restraint use questionnaire. Ryukyu Medical Journal. 2003; 22(1/2):21-8. https://jglobal.jst.go.jp/en/detail?JGLOBAL $\mathrm{ID}=200902220701831952$

[26] Chang YY, Yu HH, Loh EW, Chang LY. The efficacy of an inservice education program designed to enhance the effectiveness of physical restraints. Journal of Nursing Research. 2016; 24(1):7986. [DOI:10.1097/jnr.0000000000000092]

[27] Li X, Fawcett TN. Clinical decision making on the use of physical restraint in intensive care units. International Journal of Nursing Sciences. 2014; 1(4):446-50. [DOI:10.1016/j.jnss.2014.09.003] 
[28] McCabe DE, Alvarez CD, McNulty SR, Fitzpatrick JJ. Perceptions of physical restraints use in the elderly among registered nurses and nurse assistants in a single acute care hospital. Geriatric Nursing. 2011;32(1):39-45. [DOI:10.1016/j.gerinurse.2010.10.010]

[29] Ebadi A, Zarshenas L, Rakhshan M, Zareian A, Sharifnia SH, Mojahedi M. [Principles of scale development in health science (Persian)]. Tehran: Jame-e-Negar; 2017. http:// opac.nlai.ir/opacprod/bibliographic/4685542

[30] MacCallum RC, Widaman KF, Zhang S, Hong S. Sample size in factor analysis. Psychological Methods. 1999; 4(1):84-99. [DOI:10.1037//1082-989X.4.1.84]

[31] World Health Organization. Process of translation and adaptation of instruments. Geneva: World Health Organization; 2016. https://www.who.int/substance_abuse/research_tools/translation/en/,\%20accessed\%2020\%20September\%202016

[32] Hajizadeh E, Asghari M. [Statistical methods and analyses in health and biosciences a research methodological approach (Persian)]. Tehran: Iranian Students Booking Agency; 2011. http:// opac.nlai.ir/opac-prod/bibliographic/2069888

[33] Colton D, Covert RW. Designing and constructing instruments for social research and evaluation. San Francisco: John Wiley \& Sons; 2007. https://books.google.com/ books?id=RMMJIwx18TYC\&dq

[34] Lawshe CH. A qualitative approach to content validity. Personnel Psychology. 1975; 28(4):563-75. [DOI:10.1111/j.1744-6570.1975. tb01393.x]

[35] Waltz CF, Strickland OL, Lenz ER, editors. Measurement in nursing and health research. $4^{\text {th }}$ ed. New York: Springer Publishing Company; 2010. https://books.google.com/ books?id=1xAdjkR14ocC\&dq

[36] Polit DF, Beck CT. Essentials of nursing research: Appraising evidence for nursing practice. $8^{\text {th }}$ ed. New York: Lippincott Williams \& Wilkins; 2013. https://books.google.com/ books?id=PepTjgEACAAJ\&dq

[37] Meyers LS, Gamst GC, Guarino AJ. Applied multivariate research: Design and interpretation. Thousand Oaks, CA: SAGE Publications; 2012.

[38] Hair JF, Black WC, Babin BJ, Anderson RE.Multivariate data analysis. United States of America: Prentice Hall; 2010. https:// books.google.com/books?id=JlRaAAAAYAAJ

[39] Harrington D. Confirmatory factor analysis. New York: Oxford University Press; 2008. https://books.google.com/ books?id=PPbgH8fzwAUC\&dq 
This Page Intentionally Left Blank 